\title{
A better oxide interface?
}

1. Electrostatic carrier doping of $\mathrm{GdTiO}_{3} / \mathrm{SrTiO}_{3}$ interfaces

Authors: Pouya Moetakef, Tyler Cain, Daniel G. Ouellette, Jack Y. Zhang, Dmitri O. Klenov, Anderson Janotti, Chris G. Van de Walle, Siddharth Rajan, S. James Allen, and Susanne Stemmer

Applied Physics Letters, 99 232116, (2011).

2. Carrier-controlled ferromagnetism in $\mathrm{SrTiO}_{3}$

Authors: Pouya Moetakef, James R. Williams, Daniel G. Ouellette, Adam Kajdos, David Goldhaber-Gordon, S. James Allen, and Susanne Stemmer arXiv:1204.1081

\section{Recommendation and Commentary by Leon Balents, KITP, UCSB ${ }^{1}$}

In 2004, Ohtomo and Hwang[1] observed an electron gas at the (100) interface between two insulating oxides, $\mathrm{LaAlO}_{3}$ (LAO) and $\mathrm{SrTiO}_{3}$ (STO). They argued that this was a sign of an "electronic reconstruction" due to the different polar structures of the two materials. The LAO/STO interface has since become a subject of intense experimental and theoretical study (see for instance the JCCM commentary by Millis here: http://www.condmatjournalclub.org/?p=1452 ).

Yet some basic facts are unclear. Theory tells us that the carrier density at the interface must be "quantized" to half an electron per planar unit cell. This is essentially due to charge neutrality, and can be understand most simply by thinking of the $\mathrm{La}^{3+}$ ions as donating half an electron symmetrically to the two adjacent $\mathrm{AlO}_{2} / \mathrm{TiO}_{2}$ layers (the electrons in the $\mathrm{AlO}_{2}$ layers just complete filled shells) - see Figure. For LAO/STO, this works out to an enormous carrier density of order $3.5 \times 10^{14} \mathrm{~cm}^{-2}$. Such an ultra-high carrier density is of fundamental interest since atomic scale (Hubbard $U$ etc.) interactions amongst electrons become non-negligible in this regime. Note that this is very distinct from the strong correlations which arise at very low carrier density in semiconductor structures, which are due to the long range part of the Coulomb interaction. This density is orders of magnitude larger than what is attainable in semiconductor structures (a few times $10^{12} \mathrm{~cm}^{-2}$ ).

\footnotetext{
${ }^{1}$ Full Disclosure: the authors of papers 1,2 above are my colleagues at UCSB.
} 


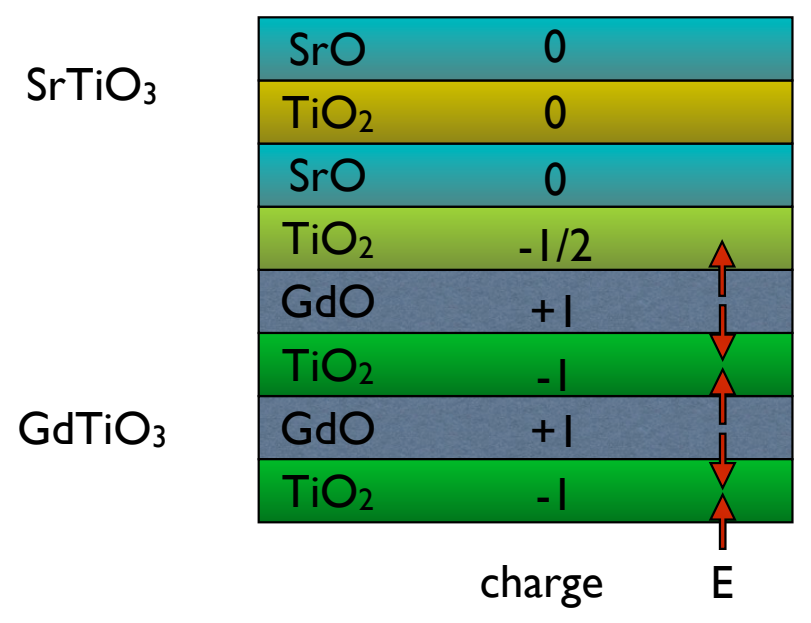

Figure 1: Schematic of charge distribution in GTO/STO (or LAO/STO) structure. Internal electric fields are shown.

Unfortunately, the carrier density observed (mainly through Hall effect) in LAO/STO is consistently at least an order of magnitude smaller than the expected value. Possible reasons for the missing carriers include "extrinsic" mechanisms such as trapping or compensation by defects, interfacial lattice deformations, etc. and "intrinsic" (i.e. interesting) phenomena such as Mott localization of electrons at the interface. New experiments on a new material have shed light on the situation. Stemmer's group at UCSB has used molecular beam epitaxy to grow heterostructures of $\mathrm{GdTiO}_{3}$ (GTO) and STO. Since GTO has the same structure as LAO and the corresponding atoms have the same ionic charge, the polar discontinuity argument applies equally as well to GTO/STO as LAO/STO (see Figure). Paper 1 above reports transport measurements on a large collection of GTO/STO structures with varying thicknesses of GTO and STO, and multiple repeats, showing a consistent appearance of $3.5 \times 10^{14} \mathrm{~cm}^{-2}$ (electron-like) carriers per interface. The carriers are argued to reside in the STO, based on their high mobility, which is insensitive to the thickness of the GTO but does decrease when the STO thickness is below a few nms.

Thus the "electronic reconstruction" driven by polar discontinuity seems to be realized in this system. This fact suggests that the reduced carrier concentration in LAO/STO is not intrinsic, but instead electrons are missing for reasons of disorder and defects. Perhaps it is natural that GTO/STO, 
in which the only difference between the two sides of the interface is the substitution of $\mathrm{Gd}$ for $\mathrm{Sr}$, is less susceptibility to defects? In any case, the GTO/STO system provides a new model system whose "good behavior" should make it more amenable to theoretical understanding. Taken as a proof of principle, it also sheds doubts on theories which suppose strong Mott localization of electrons within the $\mathrm{TiO}_{2}$ layer closest to the interface.

As mentioned above, at these ultra-high carrier concentrations, electron correlation effects are expected. Indeed, more recent work by the same group (paper 2) has shown indications - hysteretic magnetoresistance - of ferromagnetism in the same type of structures. The behavior is systematically seen when the STO layers are thinner than $2 \mathrm{~nm}$, and not for thicker layers, and the authors of paper 1 suggest that it is tied to the three-dimensional electron concentration, finding a consistent correlation of the onset temperature for hysteresis with this density. Indeed, they find similar behavior in bulk La-doped STO with 3d carrier concentrations above $10^{20} \mathrm{~cm}^{-3}$, with onset temperature consistent with the trend in GTO/STO. The latter observation helps to allay the concern that the electron gas in GTO/STO may be affected by the ferrimagnetism in GTO, which itself has a Curie transition at $20 K$ in these films.

The GTO/STO system is sure to be a subject of much ongoing research and discussion, both for its own intrinsic interest and as a point of comparison for LAO/STO. One of the most interesting issues is the influence of the correlation physics of GTO, which is itself a ferrimagnetic Mott insulator in bulk, upon the interfacial electrons. So far the influence of this appears to be minimal, but it would certainly be interesting to probe spin polarization and transport in this system in this light. So far superconductivity has not been observed in GTO/STO, but perhaps it may soon be? Both theoretical work

and further experimental characterization should help shed light on oxide interfaces in the future.

\section{References}

[1] A. Ohtomo and HY Hwang. A high-mobility electron gas at the LaAlO3/SrTiO3 heterointerface. Nature, 427(6973):423-426, 2004. 